\title{
Desarrollo de la competencia comunicativa oral en inglés a través del enfoque comunicativo y el juego de roles $^{1}$
}

\author{
Lady Karolina García Benavides ${ }^{2}$ \\ Institución Educativa Pizanda Cumbitara, Colombia
}

Recepción: 15/06/2020

Evaluación: 22/06/2020

Aceptación: 27/07/2020

Artículo de Investigación-Científica

DOI: https://doi.org/10.22267/rhec.202525.86

\section{Resumen}

El presente artículo de investigación es el resultado del estudio: "Desarrollo de la producción oral del inglés a través del Enfoque Comunicativo en los estudiantes del Centro de Idiomas de la Universidad de Nariño, Sede Ipiales", se llevó a cabo con los estudiantes de IV nivel y se enfocó en demostrar que la implementación del Enfoque Comunicativo (EC) y del juego de roles favorece la Competencia Comunicativa oral (CCO) del inglés de los mencionados estudiantes.

La aplicación de los lineamientos del paradigma cualitativo, del enfoque etnográfico y de la investigación acción que orientaron el proceso investigativo, inició con la identificación del problema y culminó con la narración de historias de la cotidianidad de la ciudad de Ipiales realizada por los estudiantes. Dichas producciones que fusionaron creativamente la competencia lingüística y la competencia comunicativa fueron integradas en la cartilla English and Culture - Authentic texts, la cual constituye un importante material didáctico que puede utilizarse para enseñar lenguas extranjeras a partir de las experiencias, intereses y expectativas que hacen parte de la cotidianidad de los estudiantes. Finalmente, el estudio permitió

\footnotetext{
1 El artículo es el resultado de la investigación de maestría titulada: "Desarrollo del uso oral del inglés a través del enfoque comunicativo en los estudiantes del Centro de Idiomas de la Universidad de Nariño, sede Ipiales"

2 Docente de Inglés en la Institución Educativa Pizanda Cumbitara, Colombia. Licenciada en Humanidades Lengua Castellana e Inglés. Estudiante de la Maestría en Educación de la Universidad de Nariño. Grupo de investigación: Lenguaje y Pedagogía. Correo electrónico: karolinagarciab12@gmail.com. 내ttp://orcid.org./0000-0001-9420-7413
} 
concluir que las dificultades de los estudiantes para expresarse oralmente en una lengua extranjera, (LE) pueden disminuir por medio de la aplicación del enfoque comunicativo y del juego de roles que, a través de un ejercicio de transposición didáctica, suscita sentimientos, sensaciones y actitudes de seguridad, autonomía, imaginación y espontaneidad que se evidencian en las interacciones verbales de los estudiantes.

Palabras clave: competencia comunicativa oral; competencia lingüística; juego de roles; enfoque comunicativo.

\title{
Oral Communicative Competence development through the use of role playing and the Communicative Approach
}

\begin{abstract}
This research article is the result of the study: "Development of the oral production of English through the Communicative Approach in students from the Language Center of Universidad de Nariño in Ipiales", carried out with students of IV level and focused on demonstrating that the implementation of the Communicative Approach (CA) and role-playing favors the students' oral Communicative Competence (OCC) of English.

By following the guidelines of the qualitative paradigm, the ethnographic approach and the action research that guided the research process, it was possible the identification of the problem which ended and with the narration of stories of the daily life of the city of Ipiales carried out by the students. These productions, which creatively merged the linguistic competence and the communicative competence, were integrated into the booklet English and Culture - Authentic texts, which constitutes an important didactic material that can be used to teach foreign languages based on the experiences, interests and expectations that are part of the students' daily lives. Finally, the study allowed concluding that students' difficulties in expressing themselves orally in a foreign language (EFL) can be reduced through the use of the communicative approach and role playing which, through an exercise of didactic transposition, promotes
\end{abstract}


feelings, sensations and attitudes of security, autonomy, imagination and spontaneity that are evidenced in the students' verbal interactions.

Keywords: language teaching; communicative competence; linguistic competence; roleplaying; communicative approach.

\section{Desenvolvimento da competência comunicativa oral em inglês por meio da abordagem comunicativa e da dramatização de papéis}

\section{Resumo}

Este artigo de pesquisa é o resultado do estudo: "Desenvolvimento da produção oral de inglês através da Abordagem Comunicativa em alunos do Centro de Línguas da Universidade de Nariño, Sede de Ipiales", realizado com alunos de nível IV e com o objetivo de demonstrar que a implementação da Abordagem Comunicativa (CE) e a dramatização favorecem a Competência Comunicativa Oral (CCO) de Inglês dos alunos citados.

A aplicação das diretrizes do paradigma qualitativo, da abordagem etnográfica e da pesquisa-ação que nortearam o processo investigativo, iniciou-se com a identificação do problema e culminou com a narração de histórias do cotidiano da cidade de Ipiales realizada pela alunos. As referidas produções que fundiram de forma criativa competência linguística e competência comunicativa foram integradas à cartilha English and Culture -Authentic texts, que constitui um importante material didático que pode ser utilizado para o ensino de línguas estrangeiras com base nas experiências, interesses e expectativas que fazem parte do dia a dia dos alunos. Por fim, o estudo permitiu concluir que as dificuldades dos alunos em se expressarem oralmente em uma língua estrangeira (LE) podem diminuir com a aplicação da abordagem comunicativa e encenação que, por meio de um exercício de transposição didática, suscita sentimentos, sensações e atitudes. de segurança, autonomia, imaginação e espontaneidade que se evidenciam nas interações verbais dos alunos. 
Palavras-chave: ensino de línguas; competência comunicativa oral; competência lingüística; dramatização; abordagem comunicativa.

\section{Introducción}

En el actual mundo globalizado el aprendizaje del inglés se ha constituido en una necesidad inminente, pues éste es el idioma de la comunicación, de los negocios, de la academia, de la ciencia, de la opinión, es decir, es el vínculo que materializa la unión entre los usuarios de todas las edades y clases sociales que habitan lugares remotos que cada día son menos lejanos gracias a las tecnologías de la informática y de la comunicación.

En consonancia con lo anterior, el Centro de Idiomas de la Universidad de Nariño sede Ipiales, zona fronteriza, contribuye a la formación intercultural de sus estudiantes y propende por la cualificación de los procesos de enseñanza, aprendizaje y evaluación que realizan sus docentes, alentándolos a emprender investigaciones que aporten con materiales y estrategias novedosas que coadyuven a la adquisición de la LE.

Los resultados de la prueba diagnóstica del manejo del inglés que realiza el Centro de Idiomas, a los estudiantes en el momento de su ingreso a la universidad, evidencian que su dominio del inglés se centra en el conocimiento de estructuras gramaticales y de vocabulario aislado que no se relacionan con necesidades comunicativas reales situadas en un contexto pragmático, cultural y sociolingüístico que convierte a la lengua en un efectivo instrumento de negociación de significados y de entendimiento que logra la anhelada interacción entre los usuarios de un idioma extranjero.

En este orden de ideas, uno de los orígenes del problema es el uso de metodologías anticuadas que aún sigue aplicando el profesor de inglés, a esto se une la preponderancia del uso de la lengua materna en el contexto de aprendizaje del inglés; de igual manera, el maestro no ha logrado integrar y desarrollar de forma equitativa las competencias lingüística y comunicativa en sus estudiantes; dicha situación torna las clases en espacios tediosos y aburridores.

Lo mencionado anteriormente se sustenta en el artículo denominado: "La enseñanza del inglés en la educación básica de personas jóvenes $y$ adultas" el cual describe una situación similar a la que se vive en el Centro de Idiomas y en el grupo de estudio seleccionados: éste refiere que las estrategias pedagógicas utilizadas en la enseñanza del inglés 
están vinculadas a metodologías tradicionales que enfatizan el desarrollo de actividades didácticas rutinarias, apoyadas en la lengua oficial y en el escaso uso de una amplia variedad de recursos didácticos. ${ }^{3}$

De acuerdo con las observaciones realizadas, es necesario reemplazar los métodos tradicionales de enseñanza por otros innovadores y más efectivos como el enfoque comunicativo a través del juego de roles que generan confianza y seguridad en el estudiante y dinamiza la enseñanza del inglés, de manera que el estudiante puede comunicarse de manera real, en forma oral o escrita, tal como lo sostienen Candlin y $\mathrm{H}$. Widdowson al afirmar que: "El aprendizaje de la lengua extranjera debe estar enfocado en el desarrollo de la competencia comunicativa y lingüística". ${ }^{4}$

El presente estudio inició con una fase exploratoria que permitió identificar el problema, avanzó a otras fases como la descriptiva, la interpretativa y la de producción de material orientado al fortalecimiento de la expresión oral; de esta manera, recorrió los pasos que se requiere para conocer a fondo un problema; buscar los sustentos teóricos, contextuales, de estado del arte y metodológicos; para tener una visión global del problema y buscar la solución pertinente y necesaria, en este caso, usar los aportes del EC y del juego de roles para producir textos orales auténticos que contribuyeron significativamente a elevar la calidad de la CCO de los estudiantes del IV nivel del Centro de Idiomas de la Universidad de Nariño, sede Ipiales.

\section{Metodología}

El proceso investigativo partió de una fase preparatoria que permitió identificar el problema en el grupo de estudio seleccionado, el conocimiento del estado del arte, del contexto y la fundamentación teórica que enriqueció el estudio con aportes de autores de reconocida autoridad en temas como epistemología de la competencia comunicativa oral en inglés, el EC, juego de roles, interdisciplinariedad, trasposición didáctica, entre otros.

3 Ricoy, María y Álvarez Sabela, "La enseñanza del inglés en la educación básica de personas jóvenes y adultas", Revista mexicana de investigación educativa, vol. 21 no. 69. (2016): 31-35, http://www.scielo.org.mx/scielo.php?script=sci arttext\&pid=S1405-66662016000200385.

4 Citado por Centro Virtual Cervantes, "El Enfoque Comunicativo", https://cvc.cervantes. es/ensenanza/biblioteca_ele/diccio_ele/diccionario/enfoquecomunicativo.htm. (Consultado el 23 de abril de 2020) 
Después de obtener el consentimiento firmado de la institución y de los padres de familia del grupo de estudio, fue posible avanzar al análisis y a la articulación de la fundamentación teórica relacionada con los objetivos, la metodología del trabajo y la estructuración de la cartilla English and Culture-authentic texts- que hicieron parte de la fase descriptiva.

La cartilla English and Culture -authentic texts es un compendio de los textos orales producidos por los estudiantes en los cuales narran historias y refieren situaciones relacionadas con la realidad y cotidianidad de la ciudad de Ipiales. Su creación se enmarca en las nuevas concepciones de la lengua que definen al aula de clase y en general a cualquier contexto de enseñanzaaprendizaje como el escenario en el cual confluyen estudiantes de diferentes edades, procedencias, culturas, ideologías y niveles socio-económicos; por consiguiente, la interacción se realiza gracias a procesos de negociación de significados y sentidos que abarcan todo tipo de manifestaciones lingüísticas, semióticas, extralingüísticas y pragmáticas entre estudiantes $\mathrm{y}$ estudiantes-profesor.

Una vez superados los pasos anteriores, se emprendió la fase interpretativa en la cual se llevó a cabo el análisis, interpretación e inferencia de los resultados obtenidos en la encuesta aplicada a los docentes y a los estudiantes del IV nivel del Centro de Idiomas. Asimismo, una vez potencializadas las habilidades de la competencia comunicativa oral, los textos auténticos recreados por los estudiantes en sus intervenciones, se plasmaron en la cartilla English and Culture -authentic texts, la cual hace parte de las evidencias del presente estudio y se constituye en una interesante herramienta didáctica.

La investigación se enmarca en el paradigma cualitativo que de acuerdo con (Henández, Fernández y Baptista (2014); centra su atención en: "Comprender los fenómenos, explorándolos desde la perspectiva de los participantes, en un ambiente natural y en relación con su contexto". 5 En este orden de ideas, se examinaron en el ámbito escolar la interrelación social, la disposición, el juego, la motivación relacionada con la expresión y el aprendizaje del inglés que se evidenció en los estudiantes en el aula de clase y el rol del docente como facilitador de la adquisición y desarrollo de las habilidades que fortalecen la CCO.

5 Roberto Hernández, Carlos Fernández y Pilar Baptista. Metodología de la Investigación. (México: Mac Graw Hill, 2014). 255. http://observatorio.epacartagena.gov.co/wpcontent/uploads/2017/08/metodologia-de-la-investigacion-sexta-edicion.compressed. pdf. (Consultado el 24 de abril de 2020). 
El enfoque etnográfico fue propicio para el presente estudio, pues permitió la participación activa de todos los actores involucrados en el proceso de enseñanza-aprendizaje de la $\mathrm{CCO}$. Este enfoque sirve para conocer el comportamiento social a través de un estudio directo de personas o grupo de personas durante un tiempo determinado. ${ }^{6}$

Considerando que la realidad investigada constituye un problema que obstaculiza la adquisición de la CCO, emanada principalmente de la falta de didácticas y de la aplicación de enfoques novedosos que impriman interés, compromiso y motivación para aprender una lengua tan importante como el inglés; la investigación acción permitió realizar un acercamiento directo, escudriñar de manera detallada, buscar y proponer soluciones que para este caso particular, proceden de la implementación del EC y del juego de roles desde la interdisciplinariedad, situados en el contexto educativo, sociocultural y pragmático de los estudiantes de IV nivel del Centro de Idiomas de la ciudad de Ipiales.

La transmisión oral de textos auténticos que nacen del diario vivir de los estudiantes, los situó en un contexto comunicativo real que potenció su expresión verbal, convirtiéndola en espontánea, creativa y propulsora de una verdadera interacción que superó el espacio del aula de clase, para aplicarse en situaciones, necesidades y contextos comunicativos reales fuera del aula donde podrá comunicarse con seguridad, con fluidez y sin temor a equivocarse.

La población que hizo parte de este estudio estuvo conformada por los 317 estudiantes y los 7 docentes del Centro de Idiomas, sede Ipiales, de ella se seleccionó el grupo de estudio, constituido por los estudiantes del IV nivel de inglés: conformado por 20 estudiantes ( 12 mujeres y 8 hombres) y un docente del género femenino.

La técnica de acopio de la información que se utilizó fue la encuesta, esta se aplicó a los estudiantes y a los docentes que hicieron parte del grupo meta. De acuerdo con los resultados de la encuesta aplicada a los estudiantes, existen falencias en la CCO relacionadas con la falta de vocabulario, la ansiedad al participar, el desinterés y tedio a las clases de inglés y la aplicación de técnicas tradicionales de la enseñanza del inglés.

Con relación a los resultados de la encuesta aplicada a los docentes, es preciso informar que esta se realizó para indagar acerca de las estrategias

${ }^{6}$ Joaquín Montano, "Método etnográfico: características, técnicas, ejemplos", https:// www.lifeder.com/metodo-etnografico/. (Consultado el 20 octubre de 2020) 
didácticas que utilizan para desarrollar y fortalecer la CCO de los estudiantes; de esta manera, fue posible conocer si su metodología marcha de acuerdo con las necesidades y requerimientos de los estudiantes quienes piden clases activas, interesantes y que se apoyen en las herramientas de las TICS; de igual manera se detectaron los vacíos y las falencias que finalmente fueron resueltas a partir de una estrategia didáctica cristalizada en la cartilla English and Culture -authentic texts-.

\section{Planteamiento del problema}

La enseñanza de los idiomas extranjeros y particularmente el inglés, ha sido objeto de rigurosas investigaciones que han permitido reorientar los enfoques, la metodología, la didáctica, la pedagogía y las técnicas, herramientas y materiales de enseñanza-aprendizaje y evaluación. No obstante, algunos docentes continúan aplicando metodologías poco eficientes que ya han sido reemplazadas por otras cuya calidad y efectividad han sido ampliamente demostradas.

La adquisición de una LE implica el desarrollo de cuatro habilidades básicas: hablar, leer, escribir y escuchar, el dominio de estas habilidades requiere de la fusión de la competencia lingüística y de la competencia comunicativa, es decir, no sólo es necesario de acuerdo con la competencia lingüística poseer vocabulario de la lengua extranjera, dominar su gramática, sintaxis, ortografía, fonología y manejar las curvas tonales para expresar diferentes significados; también es necesario, conocer la función y el significado de las palabras, de las frases y de las expresiones de una lengua de acuerdo con la sociedad y la cultura en que se usan, las funciones comunicativas que desempeña la lengua dependiendo del contexto, los usuarios y la intención comunicativa, entre otras.

Lo referido constituye el objeto del presente estudio y es el resultado de un proceso de investigación que finalmente demostró que las debilidades evidenciadas en la CCO de los estudiantes, encuentran su origen en la persistencia del uso de métodos tradicionales de enseñanza de LE y en la ausencia de estrategias de enseñanza, vacío que, según los docentes, se justifica en el desempeño deficiente de los estudiantes y en la falta de recursos en la institución educativa.

Es importante mencionar que el gobierno nacional, consciente del bajo nivel del inglés de los estudiantes que revelan las pruebas nacionales y del 
rol relevante del docente, en el proceso de enseñanza-aprendizaje de la LE, ha hecho esfuerzos ingentes para capacitarlo y actualizarlo; no obstante, la calidad del nivel de la asignatura del inglés en las distintas instituciones educativas no ha tenido una mejora sustancial; sobre el particular, Fernández (2014), manifiesta que: "Una vez que el estudiante ha tomado conciencia de su rol en el cumplimiento de sus tareas, al docente le compete organizar de manera coherente las tareas para ayudar al estudiante a desarrollar sus competencias comunicativas". ${ }^{7}$

En este orden de ideas, es vital elevar la calidad de la formación de los educadores, corregir y mejorar sus actitudes y aptitudes en su campo laboral, crear en ellos una cultura idiomática que les motive a conocer y a dominar el idioma para socializar de manera coherente y competente los conocimientos con los estudiantes, por medio de una pedagogía adecuada y de materiales didácticos que refuerzan y complementan la enseñanzaaprendizaje y evaluación; además, deben ser capaces de adecuar la enseñanza al contexto que se les presente en el desarrollo de su profesión. Por lo tanto, la responsabilidad de las instituciones educativas es la de exigir la cualificación y el desarrollo profesional continuo de sus docentes.

Por otro lado, de acuerdo con el Marco Común Europeo de referencia, es preciso considerar que la adquisición de una lengua extranjera, no sólo depende del dominio de las habilidades fonéticas, fonológicas, léxicas, gramaticales y morfológicas del estudiante, sino que en gran medida está relacionada con factores individuales como la personalidad, los intereses, las motivaciones, los valores, las creencias y los estilos cognitivos de los estudiantes y otros móviles de tipo social. ${ }^{8}$ Bajo esta perspectiva, es preciso anotar que las razones de tipo social influyen en la CCO de los estudiantes, quienes se caracterizan por ser callados y tímidos.

Los factores sociales están condicionados por las oportunidades que se presentan para aprender una lengua extranjera y los motivos, intereses y necesidades que impulsan al estudiante a aprovecharlas, con la mirada puesta en la movilidad social y en un mejor nivel de vida. En este sentido,

7 María Fernández. "La enseñanza de didáctica de lenguas extranjeras para futuros maestros no especialistas: una experiencia en Educación Primaria”. Glosas Didácticas, (11), (2004), 143-154. https://www.um.es/glosasdidacticas/doc-es/11 fernandez.pdf (Consultado el 25 de abril de 2020).

8 Centro Virtual Cervantes. "Marco Común Europeo de Referencia". https://cvc. cervantes.es/ensenanza/biblioteca_ele/diccio_ele/diccionario/enfoquecomunicativo. htm. (Consultado el 23 de abril de 2020) 
la Universidad de Nariño, ofrece la oportunidad de adquirir el idioma inglés; el contexto, por ser Ipiales una ciudad fronteriza, lo demanda, pero, muchas veces los ambientes de aprendizaje, la metodología y los materiales didácticos, desmotivan al estudiante.

Al considerar el aspecto cognitivo, se observa que la aptitud, la inteligencia, las estrategias de aprendizaje, los estilos cognitivos determinan el éxito del proceso de adquisición de una LE; desafortunadamente, tanto el proceso de enseñanza-aprendizaje, la evaluación, las explicaciones y el maestro, se expresan mediante un discurso pedagógico descontextualizado, además, se ignoran las diferencias individuales de los estudiantes que exigen variar los métodos, los materiales y los canales en que se comunica el conocimiento.

Por su parte, los factores afectivos que están relacionados con la autoestima, la inhibición, la empatía, las limitaciones, las ganas, el interés por aprender un nuevo idioma, hacen parte de las principales debilidades y dificultades que revela la prueba diagnóstica aplicada a los estudiantes cuando ingresan a la universidad: se evidenció falta de motivación hacia el aprendizaje de un nuevo idioma, bajo rendimiento académico y deserción estudiantil. Con relación a este tema, Pizarro y Josephy (2010) consideran que: "El estado emocional de los alumnos y sus actitudes actúan como un filtro, el cual permite que ingrese la información necesaria para la comprensión, o bien puede impedir o bloquear la información necesaria para la adquisición del idioma. Por otro lado, si la autoestima es baja, hay mucha ansiedad y poca participación es posible que el estudiante fracase en su proceso de aprendizaje; por el contrario, un filtro afectivo permite mejores niveles de adquisición y aprendizaje de una segunda lengua". ${ }^{9}$

\section{Resultados}

La implementación de las actividades, las técnicas y las estrategias de enseñanza-aprendizaje y evaluación que requiere el EC y el juego de roles, se llevó a cabo por medio de clases que iniciaron con un juego, la audición de una canción, la visualización de un video, entre otras actividades, continuaron con la intervención de los estudiantes en la que expresan su

9 Pizarro y Josephy, "El efecto del filtro afectivo en el aprendizaje de una segunda lengua". Letras, no 48, (2010), 13. https://www.revistas.una.ac.cr/index.php/letras/ article/view/5619, (Consultado el 23 de abril de 2020). 
opinión frente al tema, los personajes, situaciones y aspectos socioculturales que se captaron en el juego, la canción, el video o la dramatización; posteriormente, estas situaciones comunicativas son usadas para introducir y desarrollar el tema motivo de aprendizaje; finalmente, las clases cierran con una tarea comunicativa que los estudiantes realizan individualmente $o$ en grupo en contextos comunicativos reales presentes en su cotidianidad.

La puesta en acción de las actividades del EF como se anotó, están orientadas al fortalecimiento de la competencia comunicativa oral, entre las metas alcanzadas, acompañadas de las opiniones de los docentes, se destacan las siguientes:

Cuando se interrogó a los estudiantes si las clases del nivel IV cumplieron con sus expectativas sobre el desarrollo de la CCO, casi la totalidad de ellos respondió afirmativamente, sólo el $20 \%$ no creen que se hayan cumplido, las razones que aducen es que para mejorarla necesitaban trabajar con base en las estructuras gramaticales del inglés. De esta situación, se infiere que los estudiantes están acostumbrados a aprender por medio de métodos tradicionales como el de gramática y traducción. En oposición a los métodos tradicionales de enseñanza de lenguas extranjeras, Pasquali (1972), destaca el proceso de interacción en el que dos o más sujetos comparten experiencias, actúan con sentido de comunidad en función de un diálogo orientado a la construcción de conocimiento. ${ }^{10}$ Dicha acción es impulsada por la docente investigadora, cuando motiva a los estudiantes a participar en la creación de textos orales auténticos con la implementación del juego de roles.

Las respuestas a la tercera pregunta de la encuesta aplicada a los estudiantes: ¿Crees que el inglés es una asignatura importante en tu vida? ¿Por qué? Evidencian que, aunque los estudiantes refieren diferentes argumentos sobre la importancia del inglés, todos concuerdan en que la adquisición de dicho idioma es trascendental en sus proyectos de vida. Lo expresado corrobora el propósito de este estudio que propone programar actividades que requieran de la participación e interacción de los estudiantes para promover el desarrollo de la CCO en inglés. De acuerdo con lo que expresa Hymes (1974), citado por Rincón, es necesario desarrollar la habilidad para utilizar la $\mathrm{CCO}$, esto es posible a través de la experiencia

${ }^{10}$ Antonio Pasquali, Comunicación y cultura de masas (Caracas: Monte Ávila Editores, 1972), 42-56. http://www.cervantesvirtual.com/nd/ark:/59851/bmc0969884. (Consultado el 15 de abril de 2020) 
e interacción social, la acción contextualizada y la motivación ${ }^{11}$ que crea la docente investigadora cuando producen textos auténticos, acciones que conllevan a un aprendizaje efectivo de una LE. De igual manera, las exigencias del actual mundo globalizado y el deseo de superación de los estudiantes, entre otras razones, convierten el inglés en una asignatura a la que se debe dar prioridad.

El 90\% de los estudiantes que hacen parte del grupo meta, expresó su agrado por las actividades programadas por la docente investigadora y por la nueva metodología, debido a que compararon el desarrollo de las clases en las que se implementó el EC y el juego de roles, con las clases anteriores basadas en las metodologías tradicionales. De lo anterior se puede deducir que los educandos valoran significativamente el énfasis en el desarrollo del componente oral de la LE, lo que confirma que la implementación del EC y el juego de roles, es una alternativa propicia y necesaria para desarrollar la CCO en inglés que se caracteriza por ser fluida, precisa y comprensible.

El objetivo de la siguiente pregunta era conocer el grado de importancia que los estudiantes le conceden a la adquisición de la CCO y de la gramática: el 55\% de los estudiantes destacan la importancia de la CCO, mientras el $45 \%$ considera que tanto la producción oral, como la gramática son relevantes en la comunicación. Cabe precisar que, el presente estudio no pretende desconocer la importancia que tiene la competencia lingüística, es decir, la gramática, la morfología, la fonética, la fonología, la semántica, la ortografía y la habilidad ortoépica de las lenguas en la adquisición y fortalecimiento de la CCO. Por el contrario, esta es fundamental y se torna especialmente significativa cuando se la contextualiza, cuando se usan las situaciones comunicativas cotidianas como un pretexto para aprender y aplicar la competencia lingüística desde un enfoque más funcionalista que estructuralista.

Para fortalecer las anteriores apreciaciones, es pertinente acudir a lo que sostiene Lomas (2001), sobre la importancia que tiene compartir experiencias con otras personas en contextos diversos, para adquirir y dominar los conocimientos lingüísticos y textuales, para que el estudiante entienda y sienta lo que expresa de manera verbal y escrita, pues la gramática posibilita una comunicación adecuada y coherente ${ }^{12}$.

${ }^{11}$ Hymes "Hacia etnografías de la comunicación"

${ }^{12}$ Carlos Lomas, Cómo enseñar a hacer cosas con las palabras. teoría y práctica de la educación lingüística, (Barcelona: Paidós Ibérica, 2001), 34. 
Corresponde ahora, hacer referencia a las apreciaciones recibidas de los docentes, cuando se trató de recabar información sobre las estrategias que utilizan en sus clases para potenciar la $\mathrm{CCO}$ de sus estudiantes:

El $60 \%$ de los docentes encuestados prefieren profundizar y ampliar los conocimientos y las habilidades relacionadas con la producción oral del inglés, mientras que el $40 \%$ conceden importancia a la producción escrita y tienen claro que la escritura también es una habilidad fundamental en el aprendizaje de una LE; ellos aclaran que el rol de la gramática por sí sola no es suficiente y que es necesario capacitar al estudiante para que sea capaz de construir y entender oraciones.

Es evidente que la mayoría de los docentes, manejan el concepto de estrategia didáctica y la consideran importante en la planeación y desarrollo de sus clases; asimismo, son conscientes de que el logro de los objetivos de aprendizaje depende de la selección de las herramientas adecuadas; no obstante, es claro que lo que prima es la preocupación por el logro de los objetivos del curso, en lugar de la manera en que el educando se apropia del conocimiento.

Con relación a las metodologías que emplean los docentes en las clases, se encontró que prevalece el uso de métodos tradicionales y una concepción obsoleta del papel del docente, que lo concibe como el elemento más importante del proceso y como el poseedor del conocimiento, relegando al estudiante al rol de receptor pasivo que no posee conocimientos previos. Es preciso desarraigar dicha concepción y reemplazarla por los nuevos paradigmas que convierten al estudiante en protagonista del proceso y al docente en guía y orientador, que evita el transmisionismo y enseña a reflexionar de manera crítica, a desarrollar el pensamiento propio, a destacar y a valorar su identidad sociocultural, entre otros.

Sobre el uso de estrategias de enseñanza-aprendizaje, la mayoría de los docentes emplean diferentes estrategias como el aprendizaje cooperativo, el juego de roles, la conversación y en general la lúdica, además, expresan su acuerdo aobre el uso del EC para mejorar la CCO. Desafortunadamente, las respuestas de otros docentes dan a entender que no usan estrategias en sus clases y lo justifican en el desempeño de los estudiantes y en los escasos recursos que ofrece la universidad, ellos confunden los términos estrategia didáctica, técnica, recurso y actividad; por consiguiente, como es de esperarse, son los perpetuadores de los métodos tradicionales de enseñanza de la LE y de la memorización de listas infinitas y aisladas de vocabulario. 


\section{Conclusiones}

Es menester destacar la importancia que los estudiantes le conceden al aprendizaje del inglés en su proyecto de vida, en sus expectativas, intereses y necesidades. Para ellos también es relevante aprender en clases dinámicas, como las que se logran a través de la implementación del EC y del juego de roles en las cuales interactúan con sus compañeros sobre las situaciones que viven en su entorno escolar, familiar, social y cultural; pues de esta manera, es posible comunicarse en inglés de manera espontánea, fluida y sin temor a hacer el ridículo.

La aplicación del EC, permitió considerar las diferencias individuales, la dimensión afectiva y emocional, los estilos de aprendizaje, la motivación, las transacciones que se llevan a cabo dentro y fuera del aula y los factores interculturales de los estudiantes que conformaron el grupo meta, pues está demostrado que la comunicación verbal es un gran reto que aún no se ha conquistado completamente en la lengua materna y por lo tanto, representa un desafío mayor en una lengua extranjera.

La producción y uso de material didáctico como el que resultó del proceso de escritura de textos auténticos, producidos por los estudiantes del IV nivel de inglés de la Universidad de Nariño, fortaleció la CCO porque dichos textos emergieron de las experiencias que los estudiantes viven en su contexto sociocultural, porque amalgaman de manera artística varios conocimientos con una visión interdisciplinaria, porque son capaces de cristalizar una verdadera transposición didáctica. Es así como la cartilla English and Culture - Authentic texts que reúne los textos creados por los estudiantes, se convirtió en una interesante estrategia didáctica que logró una CCO clara y natural.

El aprendizaje de un idioma como el inglés, permite conocer otras culturas; ver el mundo de forma diferente; obtener beneficios personales y profesionales, pero, es un proceso laborioso que requiere del concurso de todos los involucrados en el proceso de enseñanza-aprendizaje y evaluación; con esto en mente, no está por demás insistir en la necesidad de que el maestro de lenguas extranjeras se enriquezca y actualice con el conocimiento, las prácticas y metodologías variadas que le ofrecen la academia y los institutos especializados en la enseñanza de lenguas extranjeras. 


\section{Referencias}

Centro Virtual Cervantes. "El Enfoque Comunicativo", https://cvc.cervantes.es/ ensenanza/biblioteca_ele/diccio_ele/diccionario/enfoquecomunicativo. htm. (Consultado el 23 de abril de 2020).

Fernández María. "La enseñanza de didáctica de lenguas extranjeras para futuros maestros no especialistas: una experiencia en Educación Primaria". Glosas Didácticas, (11), (2004), 143-154. https://www.um.es/glosasdidacticas/ doc-es/11 fernandez.pdf (Consultado el 25 de abril de 2020).

Hymes Dell. "Hacia etnografías de la comunicación”. México: Antología de estudios de etnolingüística y sociolingüística, 1974, 100. (Consultado el 20 de abril de 2020).

Lomas Carlos. Cómo enseñar a hacer cosas con las palabras. Teoría y práctica de la educación lingüística. Barcelona: Paidós Ibérica, 2001.

Ministerio de Educación Nacional. Bilingüismo: estrategia para la competitividad. Disponible en http:// www.mineducacion.gov.co/1621/article-97497.html. (Consultado el 10 de abril de 2020).

Montano Joaquin. "Método etnográfico: características, técnicas, ejemplos", https://www.lifeder.com/metodo-etnografico/. (Consultado el 30 octubre de 2020)

Pasquali Antonio. Comunicación y cultura de masas (Caracas: Monte Ávila Editores, 1972), 42-56. http://www.cervantesvirtual.com/nd/ark:/59851/ bmc0969884. (Consultado el 15 de abril de 2020).

Pizarro, G y Josephy D. "El efecto del filtro afectivo en el aprendizaje de una segunda lengua". Letras, no 48, (2010), 209-225. https://www.revistas. una.ac.cr/index.php/letras/article/view/5619, (Consultado el 23 de abril de 2020).

Ricoy, María y Álvarez Sabela, “La enseñanza del inglés en la educación básica de personas jóvenes y adultas" vol. 21 no. 69 (2016):31-35. http://www.scielo. org.mx/scielo.php?script=sci_arttext\&pid=S1405-66662016000200385. (Consultado el 24 de abril de 2020). 
Hernández Roberto; Fernández Carlos y Baptista Pilar. Metodología de la Investigación. México: Mac Graw Hill, 2014. 600. http://observatorio. epacartagena.gov.co/wp-content/uploads/2017/08/metodologia-de-lainvestigacion-sexta-edicion.compressed.pdf. (Consultado el 24 de abril de 2020)

Sprachcaffe, ¿Porqué estudiar inglés?. Disponible en: http://www.sprachcaffe. $\mathrm{com} /$ espanol/porque-estudiar-ingles.htm

\section{Citar este artículo}

García Benavides, Lady Karolina. "Desarrollo de la competencia comunicativa oral en inglés a través del enfoque comunicativo y el juego de roles". Revista Historia de la Educación Colombiana. Vol. 25 No 25 (2020):221-236. DOI: https://doi.org/10.22267/rhec. 202525.86 